\title{
ER stress and unfolded protein response in amyotrophic lateral sclerosis - a controversial role of protein disulphide isomerase
}

\author{
Merja Jaronen ${ }^{1,2}$, Gundars Goldsteins ${ }^{1}$ and Jari Koistinaho ${ }^{1}{ }^{*}$ \\ ${ }^{1}$ Department of Neurobiology, A. I. Virtanen Institute for Molecular Sciences, University of Eastern Finland, Kuopio, Finland \\ ${ }^{2}$ Center for Neurologic Diseases, Brigham and Women's Hospital, Harvard Medical School, Boston, MA, USA
}

\section{Edited by:}

Pier Giorgio Mastroberardino,

Erasmus MC University Medical

Center Rotterdam, Netherlands

Reviewed by:

Diego Ruano, University of Sevilla, Spain

Katia Aquilano, University of Rome Tor Vergata, Italy

${ }^{*}$ Correspondence:

Jari Koistinaho, Department of

Neurobiology, A. I. Virtanen Institute for Molecular Sciences, University

of Eastern Finland, P. O. Box

1627, Neulaniementie 2, Kuopio

70211, Finland

e-mail: jari.koistinaho@uef.fi
Accumulation of proteins in aberrant conformation occurs in many neurodegenerative diseases. Furthermore, dysfunctions in protein handling in endoplasmic reticulum (ER) and the following ER stress have been implicated in a vast number of diseases, such as amyotrophic lateral sclerosis (ALS). During excessive ER stress unfolded protein response (UPR) is activated to return ER to its normal physiological balance. The exact mechanisms of protein misfolding, accumulation and the following ER stress, which could lead to neurodegeneration, and the question whether UPR is a beneficial compensatory mechanism slowing down the neurodegenerative processes, are of interest. Protein disulphide isomerase (PDI) is a disulphide bond-modulating ER chaperone, which can also facilitate the ER-associated degradation (ERAD) of misfolded proteins. In this review we discuss the recent findings of ER stress, UPR and especially the role of PDI in ALS.

Keywords: ALS, ER stress, oxidative stress, neurodegeneration, motoneuron, glia

\section{INTRODUCTION}

Intracellular protein aggregates are characteristic of neurodegenerative diseases, including amyotrophic lateral sclerosis (ALS; Ross and Poirier, 2004), the most common motoneuron disease characterized by a selective death of upper and lower motoneurons. The exact role of protein aggregates in disease pathology is still under debate (Ross and Poirier, 2005). In ALS the majority of aggregates are ubiquitinated inclusions (Wood et al., 2003) typically containing trans-activation response element (TAR) DNA binding protein 43 (TDP-43; Neumann et al., 2006) and also mutant $\mathrm{Cu}, \mathrm{Zn}$-superoxide dismutase (SOD1) in SOD1linked familial ALS (Shibata et al., 1996) and mutant SOD1 mouse (Watanabe et al., 2001) and cell culture models (Lee et al., 2002). A likely underlying cause for protein accumulation is oxidative stress causing dissociation of SOD1 dimers to monomers and subsequently leading to aggregation (Rakhit et al., 2004). This can then together with malfunctioning proteasome degradation machinery contribute to the motoneuron dysfunction (Marino et al., 2014). Mutant SOD1s have also an ability to catalyze aberrant oxidative reactions which damages SOD1 itself (Andrus et al., 1998) possibly resulting in aggregation of mutant SOD1 (Valentine and Hart, 2003). Recent studies have further highlighted the importance of protein aggregation, especially in the case of TDP-43, in ALS pathology, as they have demonstrated that the induction of autophagy can enhance the TDP-43 turnover and neuronal survival (Barmada et al., 2014).

\section{ER STRESS AND UNFOLDED PROTEIN RESPONSE IN ALS}

As the first compartment in the secretory pathway, endoplasmic reticulum (ER) is responsible for protein synthesis, posttranslational processing, folding of newly synthesized proteins, and finally delivering the biologically active proteins to their proper target sites. The rate-limiting step in the secretory pathway is the transit to Golgi complex. If ER capacity is transcended and influx to the ER is excessive, the normal physiological state of the ER is disrupted leading to ER stress. There turn to its normal physiological balance then requires activation of the unfolded protein response (UPR) signaling pathway. If the UPR fails to restore the cell integrity, cell death signaling cascades are activated and the cell undergoes apoptosis (Schröder and Kaufman, 2005).

Changes in ER morphology have been observed in ALS patients and the G93A-SOD1 mice (Lautenschlaeger et al., 2012). G93A-SOD1 mice exhibit dilated rough ER (rER) accompanied with ribosomal detachment at preclinical and early symptomatic stages (Dal Canto and Gurney, 1995). Similar findings were demonstrated in postmortem samples of sporadic ALS (sALS) patients as researchers reported fragmentation of the rER, irregular distension of the rER cisternae and a detachment of ribosomes in degenerating anterior horn cells (Oyanagi et al., 2008). A recent study demonstrated deposits of granular or amorphous material in the ER lumen of sALS patients, indicating accumulation of misfolded proteins which could then in turn cause ER stress (Sasaki, 2010). In addition, Golgi apparatus become fragmented both in ALS patients (Fujita et al., 2000) and in G93A-SOD1 
mice (Stieber et al., 2000). Although the results indicate an early interference in the ER balance, the manifold disease course and specific disease stage of an individual cell make it difficult to draw an accurate picture of the ongoing process (Lautenschlaeger et al., 2012).

\section{CAUSES OF ER STRESS}

Accumulation of SOD1 has been considered one of the reasons for ER stress in ALS, as mutant SOD1 colocalizes with ER markers, including glucose-related protein 78 (Grp78) and calnexin (Wate et al., 2005; Kikuchi et al., 2006). Further evidence for linking UPR to SOD1 accumulation was gained by a discovery that protein disulphide isomerase (PDI), an ER chaperone, is up-regulated both in ALS patients and G93A-SOD1 mice (Atkin et al., 2006) co-localizing with accumulated mutant SOD1 (Atkin et al., 2006). Mutant SOD1 may interact with Derlin-1 and cause dysregulation of ER-associated degradation (ERAD), thereby leading to activation of ER stress-induced apoptosis signal-regulating kinase 1 (ASK1) and apoptosis (Nishitoh et al., 2008).

Another possible cause for ER stress in ALS is the imbalance of ER calcium homeostasis (Grosskreutz et al., 2010) as protein processing and folding are calcium dependent (Kuznetsov et al., 1992). Importantly, decreased ER calcium content contributes to ER stress in ALS (Jaiswal and Keller, 2009). The ER mitochondria calcium cycle hypothesis proposes that the increased calcium release from ER is coupled with calcium uptake by mitochondria and that calcium is then transported back to ER (Grosskreutz et al., 2010). This hypothesis could explain why dysfunction in the mitochondrial calcium storage (Damiano et al., 2006) would lead to disruption of the ER refilling and subsequently to ER stress and UPR (Grosskreutz et al., 2010).

\section{UNFOLDED PROTEIN RESPONSE IN ALS}

Three major ER stress sensors detect accumulation of unfolded proteins: double-stranded RNA-activated protein kinase (PKR)like ER kinase (PERK), inositol requiring enzyme 1 (IRE1), and activating transcription factor 6 (ATF6; Schröder and Kaufman, 2005). Inositol required enzyme 1 and PERK, are type I transmembrane proteins with protein kinase activity (Liu et al., 2002) while ATF6 is a type II transmembrane protein, whose cytosolic domain can translocate to the nucleus and activate UPR relevant genes (Haze et al., 1999). While the ER luminal domains of all three ER stress sensors normally bind to the ER chaperone GRP78, under ER stress GRP78 dissociates from these sensors to bind to the misfolded proteins and enables the activation of UPR sensors (Kaufman, 1999). As stated before, mutant SOD1 has been demonstrated to bind to and co-localize with GRP78, which increases its expression in ALS mice prior to the motor symptoms (Tobisawa et al., 2003).

\section{THE DOUBLE-STRANDED RNA-ACTIVATED PERK}

Dimerization and trans-autophosphorylation result in activation of the PERK kinase domain (Harding et al., 1999). Activation of PERK in turn leads to phosphorylation of the eukaryotic initiation factor-2 (eIF2 $\alpha$ ) inhibiting general translation initiation and protecting ER against an overload of newly synthesized proteins
(Harding et al., 1999). Paradoxically eIF2 $\alpha$ phosphorylation increases translation of activating transcription factor 4 ( $\mathrm{Lu}$ et al., 2004). ER kinase has also another substrate, nuclear factor erythroid 2-related factor 2 (Nrf2; Cullinan and Diehl, 2006). ER kinase pathway has been implicated in sALS (Hetz et al., 2009). Moreover, increased amounts of phospho-PERK-PERK (Atkin et al., 2006, 2008; Saxena et al., 2009) and phospho-eIF2 $\alpha$ (Saxena et al., 2009) both in G93A-SOD1 mice and Neuro2a cells transfected with mutant SOD1 have been reported.

\section{INOSITOL REQUIRING ENZYME 1 (IRE1)}

Endoplasmic reticulum stress elicits autophosphorylation of IRE1 inducing its RNAse activity (Liu et al., 2002). IRE1 mediated endoribonuclease activity consequently leads to nonconventional splicing of XBP1 (X-box binding protein 1; Calfon et al., 2002). Spliced XBP1 in turn translocates to the nucleus and controls genes related to protein quality control, protein folding, components of the ERAD pathway and genes required for lipid synthesis (Sriburi et al., 2004). Postmortem spinal cord samples from ALS and ALS mice manifest increased amounts of IRE1. Interestingly, ALS mice had augmented IRE1 amounts before the onset of symptoms (Atkin et al., 2006, 2008). Further studies have revealed up-regulation of phosphorylated IRE1 and increased amount of spliced XBP1 in G93A-SOD1 mice (Kikuchi et al., 2006). Accordingly, studies with Neuro2a cells expressing G85RSOD1 demonstrated increased splicing and nuclear translocation of XBP1 mRNA (Oh et al., 2008). The unphosphorylated and unspliced forms of IRE1 and XBP1 levels are not changed indicating that these forms could operate as an activable pool (Lautenschlaeger et al., 2012). Surprisingly, knocking down IRE1 and XBP1 by shRNA in NSC-34 cells transfected with SOD1 mutant caused decreased SOD1 aggregation and improved cell survival (Hetz et al., 2009). Augmented autophagy has been hypothesized to be the reason for the protective outcome. Generation of a knockout/transgenic mouse line by crossbreeding G86RSOD1 and XBP1 Nes-/- (Hetz et al., 2008) demonstrated several autophagic signs further strengthening the autophagy hypothesis. XBP1 Nes-/-_G86R-SOD1 mice had a slightly prolonged life span in females whereas males showed no improvement (Hetz et al., 2009).

\section{THE ACTIVATING TRANSCRIPTION FACTOR 6 (ATF6)}

Endoplasmic reticulum stress translocates ATF6 to Golgi apparatus where it is cleaved by two proteases (Haze et al., 1999). Following cleavage, the cytosolic domain of ATF6 is translocated to nucleus where it activates UPR-related genes (Gotoh et al., 2002; Yoshida et al., 2003) Elevated levels of ATF6 have been reported in ALS patients and G93A-SOD1 mice (Atkin et al., 2006, 2008). In addition, cleavage and translocation of ATF6 in Neuro2a cells transfected with mutant SOD1 has been verified (Oh et al., 2008) and knocking down ATF6 in NSC-34 cells transfected with mutant SOD1 was found to increase SOD1 aggregation (Hetz et al., 2008). Importantly, mutations in vesicle-associated membrane protein-associated protein $\mathrm{B}$ (VAPB), which have been connected with late-onset motoneuron disease, associates with intracellular membranes (Nishimura et al., 2004) as well as with UPR (Gkogkas et al., 2008). Both native and mutant 
VABP interact with ATF6 and reduce its capability to promote transcription of XBP1. The mutant VAPB is a much more potent inhibitor of ATF6 than the wild type VAPB, which may contribute to the pathological mechanisms of ALS (Gkogkas et al., 2008).

\section{CONTROVERSIAL ROLE OF PDI IN ALS}

As seen above, the majority of studies related to UPR in ALS have concentrated on motoneuronal UPR. However, damaged white matter has been reported in several neurological disorders (Matute, 2006) and, interestingly, loss of large myelinated fibers in the corticospinal tracts and ventral roots has been demonstrated in ALS patients (Underwood et al., 2011). Importantly, motoneurons that develop ER stress response are coupled with microglial activation and consequent axonal degeneration (Saxena et al., 2009). Moreover, up-regulation of UPR markers PDI and GADD34 have been demonstrated in glial cells in the spinal cord of G93A-SOD1 mice right after disease onset and shown segregation of UPR into ventral horn astrocytes and white matter microglia (Jaronen et al., 2013). This segregation is likely to reflect variable roles of UPR in astrocytes around degenerating motoneuron cell bodies and microglia around both motoneuron cell bodies and neurites. In view of the early degenerative changes in motoneuron axons and the role of microglia in front line defense, it is not surprising that microglial UPR precedes and/or dominates over astrocytic UPR during early motoneuron degeneration.

\section{PDI INACTIVATION MAY CONTRIBUTE TO PROTEIN AGGREGATION}

Protein disulphide isomerase is an enzyme of a thioredoxin superfamily primarily functioning in the ER as a chaperone protein. It facilitates the rearrangements of disulphide bonds via catalysis of thiol-disulphide exchange (Wilkinson and Gilbert, 2004; Ellgaard and Ruddock, 2005). In addition to its wellknown role in the ER, PDI has been found in other cellular localizations, such as cytosol and mitochondria, where its physiological role is not yet completely clear (Rigobello et al., 2001; Turano et al., 2002; Wilkinson and Gilbert, 2004). However, the mitochondrion-associated PDI can induce apoptosis through mitochondrial outer membrane permeabilization when accumulating at high levels in response to misfolded proteins (Hoffstrom et al., 2010). Up-regulation of PDI has been demonstrated in ALS (Atkin et al., 2006, 2008). In G93A-SOD1 rats PDI expression is increased early in the disease progression declining sharply towards the end stage (Ahtoniemi et al., 2008). The up-regulation of PDI in the early symptomatic stages of ALS (Ahtoniemi et al., 2008) might be due to the attempt to resolve the misfolding and aggregating SOD1, but as the oxidative damage increases (Goldsteins et al., 2008) PDI becomes oxidized and loses its ability to function as a disulphide bondrearranging enzyme (Figure 1). Recent report has demonstrated that S-nitrosylated and inactivated PDI can increase mutant SOD1 aggregation and trigger neuronal cell death (Jeon et al., 2014).

\section{PDI UPREGULATION MAY CAUSE NOX ACTIVATION}

Regardless of the fact that PDI is generally thought to act as a compensatory survival supporting enzyme, the other side of the PDI coin might not be as beneficial as once assumed. Recent studies have shown that in non-neuronal cells, such as vascular cells and peripheral macrophages, PDI is associated with NADPH oxidase (NOX) and act as a redox-sensitive regulatory protein of several NOX isoforms (Janiszewski et al., 2005; Laurindo et al., 2008; Santos et al., 2009a). NADPH oxidase is an enzyme that generates superoxide by transferring electrons from NADPH inside the cell across the membrane and coupling these to molecular oxygen to produce superoxide anion, a reactive free-radical. Indeed, in vivo ALS studies indicate that NOX activation and superoxide production are elevated in microglia and may contribute to motoneuron death (Wu et al., 2006). Furthermore, ER stress capable of inducing UPR has been previously shown to result in NOX activation, leading to increased superoxide production in peripheral macrophages (Li et al., 2010). As UPR has been demonstrated in microglia in the spinal cords of G93A-SOD1 mice (Jaronen et al., 2013), we hypothesize that PDI activity might be coupled to NOX-mediated reactive oxygen species (ROS) production during UPR. The view is supported by the finding that induction of UPR results in NOX activation and this activation is PDIdependent (Jaronen et al., 2013). Moreover, siRNA-mediated down-regulation of PDI expression was found to reduce NOX activation. Similar results were obtained when human primary monocytes, rat primary microglia and murine macrophage type of cells were used. These in vitro data suggest that PDI is a significant regulator of UPR-induced NOX activation in cells of hematopoietic origin. Transient expression of G93A-SOD1 inflicted augmented NOX activation in microglia BV-2 cells suggesting that mutant SOD1 is capable of triggering the UPR and finally superoxide production (Figure 1). Although several models have been proposed where PDI interacts with catalytical or regulatory subunits of NOX (Laurindo et al., 2008), the exact mechanism of how PDI activates NOX remains unclear. Based on the current knowledge PDI reductase activity may be required as bacitracin, an inhibitor of PDI reductase activity (Dickerhof et al., 2011), is able to suppress superoxide production in several cell types.

\section{EXCESSIVE PDI CATALYZED REFOLDING MAY CONTRIBUTE TO OXIDATIVE STRESS}

The main site of PDI function is the ER, where the redox conditions are very different from cytosol, enabling the protein folding. Glutathione is one of the key players in controlling the redox status of ER as it has been shown that glutathione can provide oxidizing equivalents for disulphide formation (Hwang et al., 1992). However, oxidoreductin Erol is thought to act as a primary electron acceptor in the disulphide bond formation, transferring oxidizing equivalent to its substrate PDI (Sevier et al., 2007). Ero1 oxidizes the active cysteinyl thiol groups in PDI, enabling it then in turn to oxidize the client protein and create a disulphide bond. As Erol acts as an acceptor of electrons from PDI, it passes the electrons to molecular oxygen creating harmful hydroperoxide (Figure 1; Higa and Chevet, 2012). Furthermore, reduced glutathione may be necessary for isomerization of improper disulphide bonds, resulting in oxidized glutathione (Margittai and Bánhegyi, 2010). These hydroperoxide 


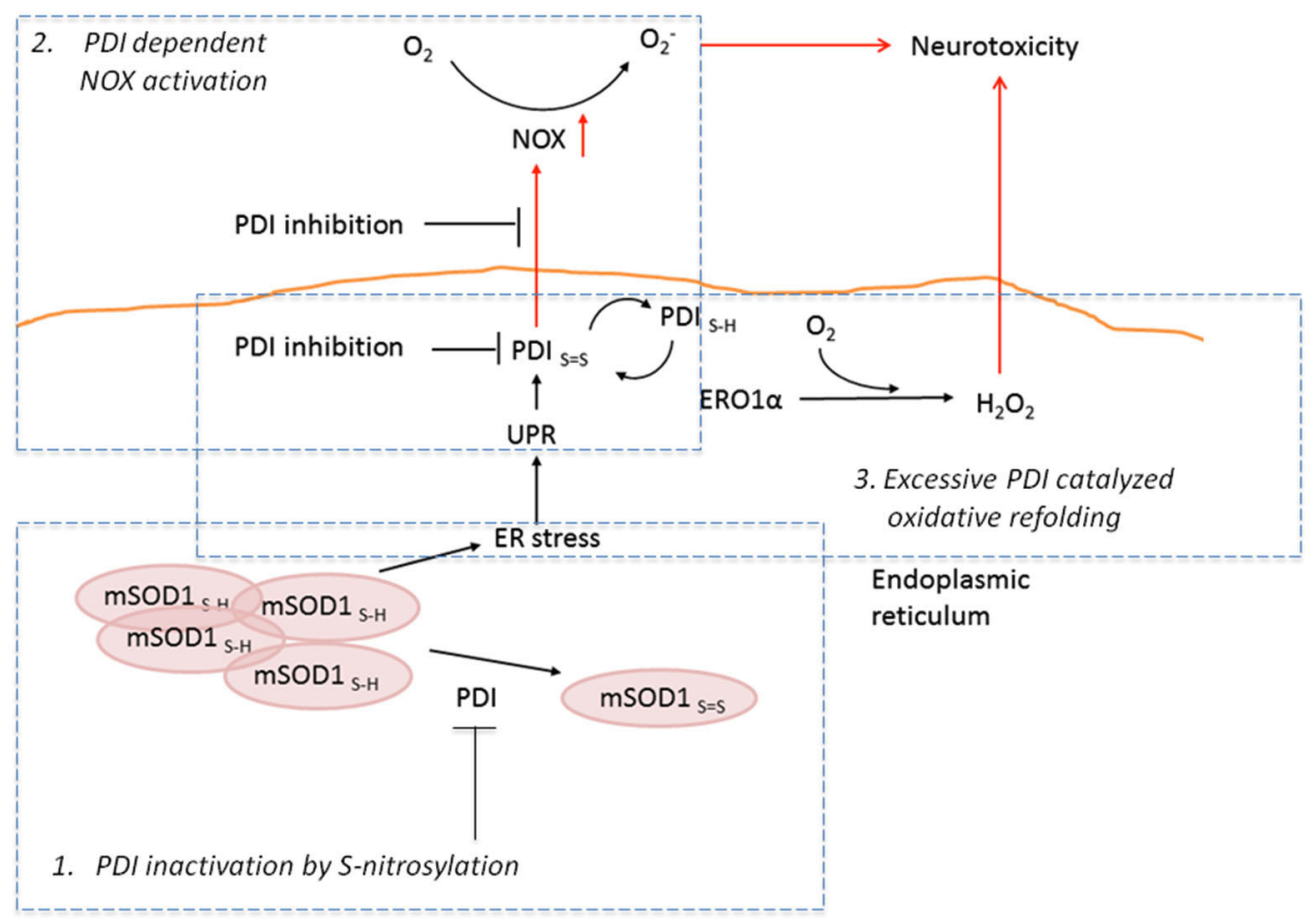

FIGURE 1 | The role of PDI in ALS. At the early stages of ALS, PDI can prevent SOD1 aggregation by re-assembling SOD1 to its active form. Protein disulphide isomerase inactivation by S-nitrosylation increases ER stress (1). Further on in the disease, the aggregation of SOD1 can lead to ER stress following UPR. Interestingly, augmented
UPR can result in increased PDI activation. This can in turn result in NOX activation, leading to increased superoxide production and finally neurotoxicity (2). The second possible route for PDI-related neurotoxicity is through $\mathrm{ERO} 1 \alpha ; \mathrm{PDI}$ activity will activate $\mathrm{ERO} 1 \alpha$, which will then in turn produce hydroperoxide and result in neurotoxicity (3). and oxidized glutathione byproducts are thought to be dangerous (Tu and Weissman, 2002; Margittai and Bánhegyi, 2010) and form a link between ER stress and oxidative stress (Harding et al., 2003; Haynes et al., 2004; Malhotra et al., 2008). However, no clear consensus on whether the Ero1-mediated extensive oxidation in the ER leads to augmented oxidative stress or acts as a part of homeostatic redox control mechanisms, has been reached (Appenzeller-Herzog, 2011). Interestingly, a recent study by Shepherd et al. (2014) shed more light over the companionship of PDI and Ero1, demonstrating that PDI has ability to catalyze both the activation and inactivation of its own catalyst Ero1.

\section{CONCLUSION}

Endoplasmic reticulum stress is a characteristic of neurodegenerative diseases, including ALS. While UPR is thought to be an adaptive and protective reaction of cells to overwhelming ER stress, the cellular response triggered by protein aggregation and UPR together may lead to misbalance in protein folding pathway and result in increased ROS production. The following increased oxidative stress upon UPR can be regarded as a union of a number of both proapoptotic and proadaptive mechanisms (Santos et al., 2009b), increased PDI expression being an integral part of the latter. However, keeping in mind that high levels of PDI in response to misfolded proteins, is also capable of promoting a cell death cascade (Hoffstrom et al., 2010), the control of PDI expression offers an interesting therapeutic strategy. In microglia cells ROS production may indeed depend on PDI, which associates with NOX and regulates its function. In agreement with our findings, recent studies have shown that overexpression of PDI promotes NOX activation in vascular smooth muscle cells (Fernandes et al., 2009). Furthermore, our notion is also supported by findings that PDI closely associates with p22phox subunit of phagocyte NOX, and that NOX activation directly correlates with PDI expression levels (Santos et al., 2009a). Currently the main scope for the role of PDI in protein aggregation linked neurodegeneration has been focused at its function in maintenance of native protein structure in neurons. Nevertheless upon excessive protein misfolding the penalty of oxidative stress originating from oxidative folding may exceed ER adaptive capabilities in neuronal cells and cause aberrant NOX activation in microglia.

\section{ACKNOWLEDGMENTS}

This project was supported by Emil Aaltonen Foundation, Waldemar von Frenckells Foundation, Sigrid Juselius Foundation and The Paulo Foundation.

\section{REFERENCES}

Ahtoniemi, T., Jaronen, M., Keksa-Goldsteine, V., Goldsteins, G., and Koistinaho, J. (2008). Mutant SOD1 from spinal cord of G93A rats is destabilized and binds to inner mitochondrial membrane. Neurobiol. Dis. 32, 479-485. doi: 10.1016/j. nbd.2008.08.010

Andrus, P. K., Fleck, T. J., Gurney, M. E., and Hall, E. D. (1998). Protein oxidative damage in a transgenic mouse model of familial amyotrophic lateral 
sclerosis. J. Neurochem. 71, 2041-2048. doi: 10.1046/j.1471-4159.1998.710 52041.x

Appenzeller-Herzog, C. (2011). Glutathione- and non-glutathione-based oxidant control in the endoplasmic reticulum. J. Cell. Sci. 124, 847-855. doi: 10.1242/jcs. 080895

Atkin, J. D., Farg, M. A., Turner, B. J., Tomas, D., Lysaght, J. A., Nunan, J., et al. (2006). Induction of the unfolded protein response in familial amyotrophic lateral sclerosis and association of protein-disulfide isomerase with superoxide dismutase 1. J. Biol. Chem. 281, 30152-30165. doi: 10.1074/jbc.m603393200

Atkin, J. D., Farg, M. A., Walker, A. K., McLean, C., Tomas, D., and Horne, M. K. (2008). Endoplasmic reticulum stress and induction of the unfolded protein response in human sporadic amyotrophic lateral sclerosis. Neurobiol. Dis. 30, 400-407. doi: 10.1016/j.nbd.2008.02.009

Barmada, S. J., Serio, A., Arjun, A., Bilican, B., Daub, A., Ando, D. M., et al. (2014). Autophagy induction enhances TDP43 turnover and survival in neuronal ALS models. Nat. Chem. Biol. 10, 677-685. doi: 10.1038/nchembio.1563

Calfon, M., Zeng, H., Urano, F., Till, J. H., Hubbard, S. R., Harding, H. P., et al. (2002). IRE1 couples endoplasmic reticulum load to secretory capacity by processing the XBP-1 mRNA. Nature 415, 92-96. doi: 10.1038/415092a

Cullinan, S. B., and Diehl, J. A. (2006). Coordination of ER and oxidative stress signaling: the PERK/Nrf2 signaling pathway. Int. J. Biochem. Cell Biol. 38, 317332. doi: 10.1016/j.biocel.2005.09.018

Dal Canto, M. C., and Gurney, M. E. (1995). Neuropathological changes in two lines of mice carrying a transgene for mutant human $\mathrm{Cu}, \mathrm{Zn}$ SOD and in mice overexpressing wild type human SOD: a model of familial amyotrophic lateral sclerosis (FALS). Brain Res. 676, 25-40. doi: 10.1016/0006-8993(95)00063-v

Damiano, M., Starkov, A. A., Petri, S., Kipiani, K., Kiaei, M., Mattiazzi, M., et al. (2006). Neural mitochondrial Ca2+ capacity impairment precedes the onset of motor symptoms in G93A Cu/Zn-superoxide dismutase mutant mice. J. Neurochem. 96, 1349-1361. doi: 10.1111/j.1471-4159.2006.03619.x

Dickerhof, N., Kleffmann, T., Jack, R., and McCormick, S. (2011). Bacitracin inhibits the reductive activity of protein disulfide isomerase by disulfide bond formation with free cysteines in the substrate-binding domain. FEBS J. 278, 2034-2043. doi: 10.1111/j.1742-4658.2011.08119.x

Ellgaard, L., and Ruddock, L. W. (2005). The human protein disulphide isomerase family: substrate interactions and functional properties. EMBO Rep. 6, 28-32. doi: 10.1038/sj.embor.7400311

Fernandes, D. C., Manoel, A. H. O., Wosniak, J. Jr., and Laurindo, F. R. (2009). Protein disulfide isomerase overexpression in vascular smooth muscle cells induces spontaneous preemptive NADPH oxidase activation and Noxl mRNA expression: effects of nitrosothiol exposure. Arch. Biochem. Biophys. 484, 197204. doi: 10.1016/j.abb.2009.01.022

Fujita, Y., Okamoto, K., Sakurai, A., Gonatas, N. K., and Hirano, A. (2000). Fragmentation of the Golgi apparatus of the anterior horn cells in patients with familial amyotrophic lateral sclerosis with SOD1 mutations and posterior column involvement. J. Neurol. Sci. 174, 137-140. doi: 10.1016/s0022$510 x(00) 00265-3$

Gkogkas, C., Middleton, S., Kremer, A. M., Wardrope, C., Hannah, M., Gillingwater, T. H., et al. (2008). VAPB interacts with and modulates the activity of ATF6. Hum. Mol. Genet. 17, 1517-1526. doi: 10.1093/hmg/ddn040

Goldsteins, G., Keksa-Goldsteine, V., Ahtoniemi, T., Jaronen, M., Arens, E., Akerman, K., et al. (2008). Deleterious role of superoxide dismutase in the mitochondrial intermembrane space. J. Biol. Chem. 283, 8446-8452. doi: 10. 1074/jbc.M706111200

Gotoh, T., Oyadomari, S., Mori, K., and Mori, M. (2002). Nitric oxide-induced apoptosis in RAW 264.7 macrophages is mediated by endoplasmic reticulum stress pathway involving ATF6 and CHOP. J. Biol. Chem. 277, 12343-12350. doi: 10.1074/jbc.m107988200

Grosskreutz, J., Van Den Bosch, L., and Keller, B. U. (2010). Calcium dysregulation in amyotrophic lateral sclerosis. Cell Calcium 47, 165-174. doi: 10.1016/j.ceca. 2009.12.002

Harding, H. P., Zhang, Y., and Ron, D. (1999). Protein translation and folding are coupled by an endoplasmic-reticulum-resident kinase. Nature 397, 271-274.

Harding, H. P., Zhang, Y., Zeng, H., Novoa, I., Lu, P. D., Calfon, M., et al. (2003). An integrated stress response regulates amino acid metabolism and resistance to oxidative stress. Mol. Cell 11, 619-633. doi: 10.1016/s1097-2765(03)00105-9

Haynes, C. M., Titus, E. A., and Cooper, A. A. (2004). Degradation of misfolded proteins prevents ER-derived oxidative stress and cell death. Mol. Cell 15, 767776. doi: 10.1016/j.molcel.2004.08.025
Haze, K., Yoshida, H., Yanagi, H., Yura, T., and Mori, K. (1999). Mammalian transcription factor ATF6 is synthesized as a transmembrane protein and activated by proteolysis in response to endoplasmic reticulum stress. Mol. Biol. Cell 10, 3787-3799. doi: 10.1091/mbc.10.11.3787

Hetz, C., Lee, A.-H., Gonzalez-Romero, D., Thielen, P., Castilla, J., Soto, C., et al. (2008). Unfolded protein response transcription factor XBP-1 does not influence prion replication or pathogenesis. Proc. Natl. Acad. Sci. U S A 105, 757-762. doi: 10.1073/pnas.0711094105

Hetz, C., Thielen, P., Matus, S., Nassif, M., Court, F., Kiffin, R., et al. (2009). XBP-1 deficiency in the nervous system protects against amyotrophic lateral sclerosis by increasing autophagy. Genes Dev. 23, 2294-2306. doi: 10.1101/gad.1830709

Higa, A., and Chevet, E. (2012). Redox signaling loops in the unfolded protein response. Cell. Signal. 24, 1548-1555. doi: 10.1016/j.cellsig.2012.03.011

Hoffstrom, B. G., Kaplan, A., Letso, R., Schmid, R. S., Turmel, G. J., Lo, D. C., et al. (2010). Inhibitors of protein disulfide isomerase suppress apoptosis induced by misfolded proteins. Nat. Chem. Biol. 6, 900-906. doi: 10.1038/nchembio.467

Hwang, C., Sinskey, A. J., and Lodish, H. F. (1992). Oxidized redox state of glutathione in the endoplasmic reticulum. Science 257, 1496-1502. doi: 10. 1126/science.1523409

Jaiswal, M. K., and Keller, B. U. (2009). Cu/Zn superoxide dismutase typical for familial amyotrophic lateral sclerosis increases the vulnerability of mitochondria and perturbs $\mathrm{Ca} 2+$ homeostasis in SOD1G93A mice. Mol. Pharmacol. 75, 478489. doi: 10.1124/mol.108.050831

Janiszewski, M., Lopes, L. R., Carmo, A. O., Pedro, M. A., Brandes, R. P., Santos, C. X. C., et al. (2005). Regulation of NAD(P)H oxidase by associated protein disulfide isomerase in vascular smooth muscle cells. J. Biol. Chem. 280, 4081340819. doi: 10.1074/jbc.m509255200

Jaronen, M., Vehviläinen, P., Malm, T., Keksa-Goldsteine, V., Pollari, E., Valonen, P., et al. (2013). Protein disulfide isomerase in ALS mouse glia links protein misfolding with NADPH oxidase-catalyzed superoxide production. Hum. Mol. Genet. 22, 646-655. doi: 10.1093/hmg/dds472

Jeon, G. S., Nakamura, T., Lee, J.-S., Choi, W.-J., Ahn, S.-W., Lee, K.-W., et al. (2014). Potential effect of S-nitrosylated protein disulfide isomerase on mutant SOD1 aggregation and neuronal cell death in amyotrophic lateral sclerosis. Mol. Neurobiol. 49, 796-807. doi: 10.1007/s12035-013-8562-z

Kaufman, R. J. (1999). Stress signaling from the lumen of the endoplasmic reticulum: coordination of gene transcriptional and translational controls. Genes Dev. 13, 1211-1233. doi: 10.1101/gad.13.10.1211

Kikuchi, H., Almer, G., Yamashita, S., Guégan, C., Nagai, M., Xu, Z., et al. (2006). Spinal cord endoplasmic reticulum stress associated with a microsomal accumulation of mutant superoxide dismutase-1 in an ALS model. Proc. Natl. Acad. Sci. U S A 103, 6025-6030. doi: 10.1073/pnas.0509227103

Kuznetsov, G., Brostrom, M. A., and Brostrom, C. O. (1992). Demonstration of a calcium requirement for secretory protein processing and export. Differential effects of calcium and dithiothreitol. J. Biol. Chem. 267, 3932-3939.

Laurindo, F. R. M., Fernandes, D. C., Amanso, A. M., Lopes, L. R., and Santos, C. X. C. (2008). Novel role of protein disulfide isomerase in the regulation of NADPH oxidase activity: pathophysiological implications in vascular diseases. Antioxid. Redox Signal. 10, 1101-1113. doi: 10.1089/ars.2007.2011

Lautenschlaeger, J., Prell, T., and Grosskreutz, J. (2012). Endoplasmic reticulum stress and the ER mitochondrial calcium cycle in amyotrophic lateral sclerosis. Amyotroph. Lateral Scler. 13, 166-177. doi: 10.3109/17482968.2011. 641569

Lee, J. P., Gerin, C., Bindokas, V. P., Miller, R., Ghadge, G., and Roos, R. P. (2002). No correlation between aggregates of $\mathrm{Cu} / \mathrm{Zn}$ superoxide dismutase and cell death in familial amyotrophic lateral sclerosis. J. Neurochem. 82, 1229-1238. doi: 10.1046/j.1471-4159.2002.01056.x

Li, G., Scull, C., Ozcan, L., and Tabas, I. (2010). NADPH oxidase links endoplasmic reticulum stress, oxidative stress and PKR activation to induce apoptosis. J. Cell Biol. 191, 1113-1125. doi: 10.1083/jcb.201006121

Liu, C. Y., Wong, H. N., Schauerte, J. A., and Kaufman, R. J. (2002). The protein kinase/endoribonuclease IRE1alpha that signals the unfolded protein response has a luminal N-terminal ligand-independent dimerization domain. J. Biol. Chem. 277, 18346-18356. doi: 10.1074/jbc.m112454200

Lu, P. D., Harding, H. P., and Ron, D. (2004). Translation reinitiation at alternative open reading frames regulates gene expression in an integrated stress response. J. Cell Biol. 167, 27-33. doi: 10.1083/jcb.200408003

Malhotra, J. D., Miao, H., Zhang, K., Wolfson, A., Pennathur, S., Pipe, S. W., et al. (2008). Antioxidants reduce endoplasmic reticulum stress and improve protein 
secretion. Proc. Natl. Acad. Sci. U S A 105, 18525-18530. doi: 10.1073/pnas. 0809677105

Margittai, E., and Bánhegyi, G. (2010). Oxidative folding in the endoplasmic reticulum: towards a multiple oxidant hypothesis? FEBS Lett. 584, 2995-2998. doi: 10.1016/j.febslet.2010.05.055

Marino, M., Papa, S., Crippa, V., Nardo, G., Peviani, M., Cheroni, C., et al. (2014). Differences in protein quality control correlate with phenotype variability in 2 mouse models of familial amyotrophic lateral sclerosis. Neurobiol. Aging. doi: 10 . 1016/j.neurobiolaging.2014.06.026. [Epub ahead of print].

Matute, C. (2006). Oligodendrocyte NMDA receptors: a novel therapeutic target. Trends Mol. Med. 12, 289-292. doi: 10.1016/j.molmed.2006.05.004

Neumann, M., Sampathu, D. M., Kwong, L. K., Truax, A. C., Micsenyi, M. C., Chou, T. T., et al. (2006). Ubiquitinated TDP-43 in frontotemporal lobar degeneration and amyotrophic lateral sclerosis. Science 314, 130-133. doi: 10.1126/science. 1134108

Nishimura, A. L., Mitne-Neto, M., Silva, H. C. A., Richieri-Costa, A., Middleton, S., Cascio, D., et al. (2004). A mutation in the vesicle-trafficking protein VAPB causes late-onset spinal muscular atrophy and amyotrophic lateral sclerosis. Am. J. Hum. Genet. 75, 822-831. doi: 10.1086/425287

Nishitoh, H., Kadowaki, H., Nagai, A., Maruyama, T., Yokota, T., Fukutomi, H., et al. (2008). ALS-linked mutant SOD1 induces ER stress- and ASK1-dependent motor neuron death by targeting Derlin-1. Genes Dev. 22, 1451-1464. doi: 10. 1101/gad.1640108

Oh, Y. K., Shin, K. S., Yuan, J., and Kang, S. J. (2008). Superoxide dismutase 1 mutants related to amyotrophic lateral sclerosis induce endoplasmic stress in neuro2a cells. J. Neurochem. 104, 993-1005. doi: 10.1111/j.1471-4159.2007. 05053.x

Oyanagi, K., Yamazaki, M., Takahashi, H., Watabe, K., Wada, M., Komori, T., et al. (2008). Spinal anterior horn cells in sporadic amyotrophic lateral sclerosis show ribosomal detachment from and cisternal distention of the rough endoplasmic reticulum. Neuropathol. Appl. Neurobiol. 34, 650-658. doi: 10.1111/j.1365-2990. 2008.00941.x

Rakhit, R., Crow, J. P., Lepock, J. R., Kondejewski, L. H., Cashman, N. R., and Chakrabartty, A. (2004). Monomeric $\mathrm{Cu}, \mathrm{Zn}$-superoxide dismutase is a common misfolding intermediate in the oxidation models of sporadic and familial amyotrophic lateral sclerosis. J. Biol. Chem. 279, 15499-15504. doi: 10.1074/jbc. m313295200

Rigobello, M. P., Donella-Deana, A., Cesaro, L., and Bindoli, A. (2001). Distribution of protein disulphide isomerase in rat liver mitochondria. Biochem. J. 356, 567-570. doi: 10.1042/0264-6021:3560567

Ross, C. A., and Poirier, M. A. (2004). Protein aggregation and neurodegenerative disease. Nat. Med. 10, S10-S17. doi: 10.1038/nm1066

Ross, C. A., and Poirier, M. A. (2005). Opinion: what is the role of protein aggregation in neurodegeneration? Nat. Rev. Mol. Cell Biol. 6, 891-898. doi: 10. $1038 /$ nrm 1742

Santos, C. X. C., Stolf, B. S., Takemoto, P. V. A., Amanso, A. M., Lopes, L. R., Souza, E. B., et al. (2009a). Protein disulfide isomerase (PDI) associates with NADPH oxidase and is required for phagocytosis of Leishmania chagasi promastigotes by macrophages. J. Leukoc. Biol. 86, 989-998. doi: 10.1189/jlb.0608354

Santos, C. X. C., Tanaka, L. Y., Wosniak, J., and Laurindo, F. R. M. (2009b). Mechanisms and implications of reactive oxygen species generation during the unfolded protein response: roles of endoplasmic reticulum oxidoreductases, mitochondrial electron transport and NADPH oxidase. Antioxid. Redox Signal. 11, 2409-2427. doi: 10.1089/ARS.2009.2625

Sasaki, S. (2010). Endoplasmic reticulum stress in motor neurons of the spinal cord in sporadic amyotrophic lateral sclerosis. J. Neuropathol. Exp. Neurol. 69, 346355. doi: 10.1097/nen.0b013e3181d44992

Saxena, S., Cabuy, E., and Caroni, P. (2009). A role for motoneuron subtypeselective ER stress in disease manifestations of FALS mice. Nat. Neurosci. 12, 627-636. doi: 10.1038/nn.2297

Schröder, M., and Kaufman, R. J. (2005). The mammalian unfolded protein response. Annu. Rev. Biochem. 74, 739-789. doi: 10.1146/annurev.biochem.73. 011303.074134

Sevier, C. S., Qu, H., Heldman, N., Gross, E., Fass, D., and Kaiser, C. A. (2007). Modulation of cellular disulfide-bond formation and the ER redox environment by feedback regulation of Ero1. Cell 129, 333-344. doi: 10.1016/j.cell.2007. 02.039
Shepherd, C., Oka, O. B. V., and Bulleid, N. J. (2014). Inactivation of mammalian Ero $1 \alpha$ is catalysed by specific protein disulfide-isomerases. Biochem. J. 461, 107113. doi: $10.1042 / \mathrm{BJ} 20140234$

Shibata, N., Asayama, K., Hirano, A., and Kobayashi, M. (1996). Immunohistochemical study on superoxide dismutases in spinal cords from autopsied patients with amyotrophic lateral sclerosis. Dev. Neurosci. 55, 481-490.

Sriburi, R., Jackowski, S., Mori, K., and Brewer, J. W. (2004). XBP1: a link between the unfolded protein response, lipid biosynthesis and biogenesis of the endoplasmic reticulum. J. Cell Biol. 167, 35-41. doi: 10.1083/jcb.200406136

Stieber, A., Gonatas, J. O., Collard, J., Meier, J., Julien, J., Schweitzer, P., et al. (2000). The neuronal Golgi apparatus is fragmented in transgenic mice expressing a mutant human SOD1, but not in mice expressing the human NF-H gene. J. Neurol. Sci. 173, 63-72. doi: 10.1016/s0022-510x(99)00301-9

Tobisawa, S., Hozumi, Y., Arawaka, S., Koyama, S., Wada, M., Nagai, M., et al. (2003). Mutant SOD1 linked to familial amyotrophic lateral sclerosis, but not wild-type SOD1, induces ER stress in COS7 cells and transgenic mice. Biochem. Biophys. Res. Commun. 303, 496-503. doi: 10.1016/s0006-291x(03)00353-x

Tu, B. P., and Weissman, J. S. (2002). The FAD- and O(2)-dependent reaction cycle of Erol-mediated oxidative protein folding in the endoplasmic reticulum. Mol. Cell 10, 983-994. doi: 10.1016/s1097-2765(02)00696-2

Turano, C., Coppari, S., Altieri, F., and Ferraro, A. (2002). Proteins of the PDI family: unpredicted non-ER locations and functions. J. Cell. Physiol. 193, 154163. doi: $10.1002 /$ jcp. 10172

Underwood, C. K., Kurniawan, N. D., Butler, T. J., Cowin, G. J., and Wallace, R. H. (2011). Non-invasive diffusion tensor imaging detects white matter degeneration in the spinal cord of a mouse model of amyotrophic lateral sclerosis. Neuroimage 55, 455-461. doi: 10.1016/j.neuroimage.2010.12.044

Valentine, J. S., and Hart, P. J. (2003). Misfolded CuZnSOD and amyotrophic lateral sclerosis. Proc. Natl. Acad. Sci. U S A 100, 3617-3622. doi: 10.1073/pnas. 0730423100

Watanabe, M., Dykes-Hoberg, M., Culotta, V. C., Price, D. L., Wong, P. C., and Rothstein, J. D. (2001). Histological evidence of protein aggregation in mutants SOD1 transgenic mice and in amyotrophic lateral sclerosis neural tissues. Neurobiol. Dis. 8, 933-941. doi: 10.1006/nbdi.2001.0443

Wate, R., Ito, H., Zhang, J. H., Ohnishi, S., Nakano, S., and Kusaka, H. (2005). Expression of an endoplasmic reticulum-resident chaperone, glucose-regulated stress protein 78, in the spinal cord of a mouse model of amyotrophic lateral sclerosis. Acta Neuropathol. 110, 557-562. doi: 10.1007/s00401-005-1080-y

Wilkinson, B., and Gilbert, H. F. (2004). Protein disulfide isomerase. Biochim. Biophys. Acta 1699, 35-44. doi: 10.1016/j.bbapap.2004.02.017

Wood, J. D., Beaujeux, T. P., and Shaw, P. J. (2003). Protein aggregation in motor neurone disorders. Neuropathol. Appl. Neurobiol. 29, 529-545. doi: 10.1046/j. 0305-1846.2003.00518.x

Wu, D.-C., Ré, D. B., Nagai, M., Ischiropoulos, H., and Przedborski, S. (2006). The inflammatory NADPH oxidase enzyme modulates motor neuron degeneration in amyotrophic lateral sclerosis mice. Proc. Natl. Acad. Sci. U S A 103, 1213212137. doi: $10.1073 /$ pnas. 0603670103

Yoshida, H., Matsui, T., Hosokawa, N., Kaufman, R. J., Nagata, K., and Mori, K. (2003). A time-dependent phase shift in the mammalian unfolded protein response. Dev. Cell 4, 265-271. doi: 10.1016/s1534-5807(03)00022-4

Conflict of Interest Statement: The authors declare that the research was conducted in the absence of any commercial or financial relationships that could be construed as a potential conflict of interest.

Received: 02 August 2014; accepted: 07 November 2014; published online: 02 December 2014.

Citation: Jaronen M, Goldsteins G and Koistinaho J (2014) ER stress and unfolded protein response in amyotrophic lateral sclerosis-a controversial role of protein disulphide isomerase. Front. Cell. Neurosci. 8:402. doi: 10.3389/fncel.2014.00402 This article was submitted to the journal Frontiers in Cellular Neuroscience. Copyright (๑) 2014 Jaronen, Goldsteins and Koistinaho. This is an open-access article distributed under the terms of the Creative Commons Attribution License (CC BY). The use, distribution and reproduction in other forums is permitted, provided the original author(s) or licensor are credited and that the original publication in this journal is cited, in accordance with accepted academic practice. No use, distribution or reproduction is permitted which does not comply with these terms. 